\title{
In-phase and Antiphase Complete Chaotic Synchronization in Symmetrically Coupled Discrete Maps
}

\author{
VLADIMIR ASTAKHOV, ALEXEY SHABUNIN*, ALEXANDER KLIMSHIN \\ and VADIM ANISHCHENKO \\ Radiophysics and Nonlinear Dynamics Department, Saratov State University, \\ Astrakhanskaya, 83, 410026, Saratov, Russia
}

(Received 21 September 2000)

\begin{abstract}
We consider in-phase and antiphase synchronization of chaos in a system of coupled cubic maps. Regions of stability and robustness of the regime of in-phase complete synchronization was found. It was demonstrated that the loss of the synchronization is accompanied by bubbling and riddling phenomena. The mechanisms of these phenomena are connected with bifurcations of the main family of periodic orbits and orbits appeared from them. We found that in spite of the in-phase synchronization, the antiphase self-synchronization of chaos is impossible for discrete maps with symmetric diffusive coupling. For achieving antiphase synchronization we used method of controlled synchronization by addition feedback. The region of the controlled antiphase synchronization and phenomena which accompany the loss of the synchronization are presented.
\end{abstract}

Keywords: Nonlinear dynamics; Chaotic synchronization; Chaos control

\section{INTRODUCTION}

At present, the synchronization of chaos is in the center of attention of many researches. There are several approaches to the term "chaotic synchronization". One of them is the complete synchronization of chaos when coupled oscillators move identically to each other $\left(x_{1}(t)=x_{2}(t)\right)$ (Fujisaka, 1983). The another case of full synchronization is when the subsystems oscillate identically but with opposite signs: $x_{1}(t)=-x_{2}(t)$. This case can be called as antiphase complete chaotic synchronization. The antiphase synchronization of chaos was considered in the work (Cao, 1998). The authors investigated "master - slave" synchronization (Pecora, 1990), when one subsystem unidirectionally influences on the other one. The possibility of chaotic antiphase self-synchronization and

*e-mail: alexey@chaos.ssu.runnet.ru

ISSN: 1026-0226 C 2002 Taylor \& Francis Ltd 
controlled antiphase synchronization in symmetrically coupled oscillators takes further consideration.

As it was shown the in-phase complete synchronization of chaos exists in the determine interval of the coupling coefficient. With changing the coupling the regime of synchronization breaks. This process can be accompanied by the bubbling phenomenon, the blowout bifurcation and the riddled basins (Ashvin, 1994; Lai, 1996). A lot of works considering characteristic bifurcations which lead to destruction of the regime of synchronous oscillations appear for the last time (Pikovsky, 1991; Astakhov, 1998). There are reasons that scenarium of loss of chaotic synchronization must be in close connection with bifurcational mechanisms which form the chaotic attractor related to the synchronous oscillations. One of the typical mechanisms for many systems is the period-doubling cascade. The chaotic attractor formed on the base of the cascade of subhurmonic bifurcations contains infinite number of saddle cycles which form the skeleton of the chaotic attractor.

In the works on investigation of synchronization in the coupled logistic maps (Astakhov, 1997; Astakhov, 1998) we found that the desynchronization of chaos is caused by bifurcations of saddle periodic orbits of the main family, on the base of which the chaotic attractor was formed. Consideration of other dynamical systems with the similar scenarium of the transition to chaos gives possibility to reveal both the community of the found laws and differences which are determined by the dynamics of particular system.

In a system of symmetrically coupled identical oscillators a limit set relating to synchronous oscillations locates in the symmetric subspace $\left(\mathbf{x}_{1}=\mathbf{x}_{2}\right)$ (for the in-phase synchronization) or in the antisymmetric subspace $\left(\mathbf{x}_{1}=-\mathbf{x}_{2}\right)$ (for the antiphase synchronization) of the whole phase space of the system, where $\mathbf{x}_{1}$ and $\mathbf{x}_{2}$ are vectors of identical dynamical variables of interacting subsystems. If the chaotic set is attractive in the normal to the subspace direction, namely when the largest normal Lyapunov exponent is neg- ative, the synchronous oscillations are observed in experiment. When the exponent changes its sign to the positive, the chaotic attractor becomes nonattracting in the normal direction and transforms to the chaotic saddle. The synchronous oscillations lose its stability and they are not observed in experiment further. However, it is possible that the largest Lyapunov exponent on the chaotic attractor is negative, but the exponents on some limit sets encapsulated in the attractor are positive. In this case the synchronous regime remains stable but becomes unrobust. Any infinitesimal noise or the parameters mismatch can lead to the bubbling of attractor. The time-series of the difference between oscillations in the subsystems $\left(x_{1}-x_{2}\right)$ is intermittency process when the phase point moves in vicinity of the symmetric subspace for a long time (laminar phase) and leave away from it from time to time (turbulent bursts) (Platt, 1993). The bubbling of attractor is the first step to the desynchronization of chaos. Then, with changing of the controlling parameters more quantity of encapsulated cycles lose their stability in the normal direction. This enforces the process of bubbling and then the averaged on the attractor highest normal Lyapunov exponent can become positive. As a result, the chaotic set in the symmetric subspace becomes nonattractive. This phenomenon is called the blowout bifurcation (Ashvin, 1994). The bubbling of attractor can be followed by the riddling of its basins when "holes" from the basins of another attractor appear in infinitesimly small vicinity of the attractor. In this case, the presence of small noise or the parameters mismatch leads to leaving of the phase point to the another attractor. Regimes which accompany the process of chaotic synchronization loss in the coupled logistic maps were described in works (Fujisaka, 1985; Kuznetzov, 1985). Mechanisms of the synchronization loss in the coupled logistic maps is described in detail in (Astakhov, 1997; Astakhov, 1998; Maistrenko, 1998; Maistrenko, 1999).

In the present work we hold bifurcational analysis of the system of coupled cubic maps and consider observable phenomena which accompany 
the investigated bifurcations. The obtained results are compared with the results for other systems. We consider the case of antiphase synchronization of chaos and demonstrate that in similar systems the self-synchronization is impossible. To achieve regime of the chaotic antiphase synchronization we apply method of feedback chaos control. We show that loss of this type of synchronization is also accompanied by the bubbling and the riddling phenomenon and by the blowout bifurcation.

\section{THE IN-PHASE}

AND THE ANTIPHASE

SYNCHRONIZATION

IN DIFFUSIVELY COUPLED MAPS

Let's consider a system of two identical discrete maps with symmetrical diffusive coupling:

$$
\begin{aligned}
& x_{n+1}=f\left(x_{n}\right)+\gamma\left(f\left(y_{n}\right)-f\left(x_{n}\right)\right) \\
& y_{n+1}=f\left(y_{n}\right)+\gamma\left(f\left(x_{n}\right)-f\left(y_{n}\right)\right)
\end{aligned}
$$

It is seen, that this system is invariant to the transformation $x \leftrightarrow y$ and therefore the subspace $(x=y)$ is invariant to the operator of the evolution of the system. For investigation stability properties of the symmetric solutions it is convenient to use "normal" variables:

$$
\begin{aligned}
& u=\frac{x+y}{2} \\
& v=\frac{x-y}{2}
\end{aligned}
$$

Adding and subtracting Eqs. (1) and (2) and then linearizing results in the vicinity of the symmetric subspace we get:

$$
\begin{gathered}
u_{n+1}=f\left(u_{n}\right) \\
v_{n+1}=(1-2 \gamma) f^{\prime}\left(u_{n}\right) v_{n}
\end{gathered}
$$

The Eq. (3) describes the dynamics inside the symmetric subspace. It is evidently the equation of the single map. The tangent stability of the synchronous solution is described by the tangent Lyapunov exponent:

$$
\Lambda_{\tau}^{i}=\lim _{N \rightarrow \infty} \frac{1}{N} \sum_{n=1}^{N} \ln \left|f^{\prime}\left(u_{n}\right)\right|
$$

The Eq. (4) describes the dynamics in the normal direction to the symmetric subspace in its small vicinity. The normal stability of the synchronous solution is described by the normal Lyapunov exponent:

$$
\Lambda_{\perp}^{i}=\lim _{N \rightarrow \infty} \frac{1}{N} \sum_{n=1}^{N} \ln \left|(1-2 \gamma) f^{\prime}\left(u_{n}\right)\right|
$$

Comparing (5) and (6) we see that the tangent and the normal Lyapunov exponents satisfy the relation:

$$
\Lambda_{\perp}^{i}=\Lambda_{\tau}^{i}+\ln |1-2 \gamma|
$$

If we use small positive coupling $(0<\gamma<0.5)$ the normal Lyapunov exponent is smaller than the tangent one. Hence, any in-phase regular oscillations are normally stable and in-phase chaotic oscillation are stable only at sufficient coupling.

Now let's consider the antiphase synchronization. In this case we'll also use normal variables. The equations in the small vicinity of the antisymmetric subspace have the form:

$$
\begin{gathered}
u_{n+1}=f^{\prime}\left(v_{n}\right) u_{n} \\
v_{n+1}=(1-2 \gamma) f\left(v_{n}\right)
\end{gathered}
$$

In this case the dynamics inside the antisymmetric subspace is described by the Eq. (9). In spite of the case of the in-phase synchronization it depends on the coupling coefficient $\gamma$. Stability of a antisymmetric solution to the tangent perturbations is determined by the tangent Lyapunov exponent:

$$
\Lambda_{\tau}^{a}=\lim _{N \rightarrow \infty} \frac{1}{N} \sum_{n=1}^{N} \ln \left|(1-2 \gamma) f^{\prime}\left(v_{n}\right)\right|
$$

The Eq. (8) determines dynamics in the normal direction to the antisymmetric subspace in its 
vicinity. It has no obvious dependence on the coupling coefficient $\gamma$ but it depend on it through the variable $v_{n}$, which is determined by the Eq. (9). The normal Lyapunov exponent which determines transversal stability of the antiphase oscillations has the form:

$$
\Lambda_{\perp}^{a}=\lim _{N \rightarrow \infty} \frac{1}{N} \sum_{n=1}^{N} \ln \left|f^{\prime}\left(v_{n}\right)\right|
$$

It is seen that the normal and tangent Lyapunov exponents are connected with each other:

$$
\Lambda_{\tau}^{a}=\Lambda_{\perp}^{a}+\ln |1-2 \gamma|
$$

This relation is the opposite to the in-phase case. Here $\Lambda_{\tau}^{a} \leq \Lambda_{\perp}^{a}$ and hence the every antiphase oscillating regime firstly loses its stability in the normal to the antisymmetric subspace direction and secondly in the tangent direction. Because of relation (12) the antiphase self-synchronization of chaos is impossible in the considered systems. For a chaotic attractor $\Lambda_{\tau}^{a}>0$ and therefore the normal Lyapunov exponent must be positive. Hence chaotic antiphase oscillations couldn't be transversally stable.

For stabilization of antiphase chaotic oscillations it is possible to use feedback controlling influence. We wanted to find the controlling function in the form which does not change the form of antiphase oscillations, but changes their stability. Hence, the controlling function $\Psi(x, y)$ must be equal to zero inside the antisymmetric subspace, namely: $\Psi(x,-x)=0$. In our work we suggest the function in the form: $\Psi(x, y)=r(f(x)+f(y))$. The controlling term is added to the right side of the first equation of the system (1), (2):

$$
\begin{gathered}
x_{n+1}=f\left(x_{n}\right)+\gamma\left(f\left(y_{n}\right)-f\left(x_{n}\right)\right)+r\left(f\left(x_{n}\right)+f\left(y_{n}\right)\right) \\
y_{n+1}=f\left(y_{n}\right)+\gamma\left(f\left(x_{n}\right)-f\left(y_{n}\right)\right)
\end{gathered}
$$

The term $r\left(f\left(x_{n}\right)+f\left(y_{n}\right)\right)$ can be considered as another coupling loop with coupling coefficient $r$.
The equations in the normal variables for the system with the control have the form (near the antisymmetric subspace):

$$
\begin{gathered}
u_{n+1}=(1+r) f^{\prime}\left(v_{n}\right) u_{n} \\
v_{n+1}=(1-2 \gamma) f\left(v_{n}\right)+r f^{\prime}\left(v_{n}\right) u_{n}
\end{gathered}
$$

In the case of antiphase oscillations $u_{n}=0$ and Eq. (16) transforms to the (9). The normal Lyapunov exponent for the system with the control is:

$$
\Lambda_{\perp \text { contr }}^{a}=\lim _{N \rightarrow \infty} \frac{1}{N} \sum_{n=1}^{N} \ln \left|(1+r) f^{\prime}\left(v_{n}\right)\right|
$$

and hence:

$$
\Lambda_{\perp \text { contr }}^{a}=\Lambda_{\perp}+\ln |1+r|
$$

Choosing $r$ sufficiently close to -1 , we can achieve any small value for the normal Lyapunov exponent and hence can stabilize antiphase oscillations.

\section{THE SYSTEM UNDER CONSIDERATION}

Let's consider a system of diffusivelly coupled cubic maps in the form:

$$
\begin{aligned}
& x_{n+1}=f\left(x_{n}\right)+\gamma\left(f\left(y_{n}\right)-f\left(x_{n}\right)\right) \\
& y_{n+1}=f\left(y_{n}\right)+\gamma\left(f\left(x_{n}\right)-f\left(y_{n}\right)\right),
\end{aligned}
$$

where $f(x)=(a-1) x-a x^{3}, a$ is the parameter of the subsystem, $\gamma$ is the coefficient of coupling.

The single cubic map:

$$
x_{n+1}=(a-1) x_{n}-a x_{n}^{3}
$$

has a symmetry to transformation of the coordinate:

$$
I: x \leftrightarrow-x,
$$

Therefore all its limit sets are self-symmetric to this transformation or they have symmetric ones. 
At any values of the parameter $a$ in the phase space of the system there is a period-one fixed point. At $0<a<2$ this point is stable, at $a=2$ its multiplier $\mu=a-1$ becomes equal to 1 . As a result the point becomes unstable and in its vicinity a pear of symmetric to each other period-one points appears:

$$
C_{1}: x=\sqrt{\frac{a-2}{a}} C_{2}: x=-\sqrt{\frac{a-2}{a}} .
$$

With increasing of the parameter $a$ a cascade of the period-doubling bifurcations takes place on the base of these points $C_{1}$ and $C_{2}$ which leads to formation of chaotic attractors. At $a=3.6$ they merge to a single attractor.

A system of the coupled maps posses the symmetric property of the single map to the transformation:

$$
I: x \leftrightarrow-x, y \leftrightarrow-y,
$$

and due to the symmetric coupling and identity of the subsystems it also posses symmetry to transformation:

$$
R: x \leftrightarrow y .
$$

Because $I$ and $R$ commutate with each other, their combination is also a symmetric transformation for the system (1):

$$
I \circ R: x \leftrightarrow-y, y \leftrightarrow-x .
$$

Consequence of the symmetry of the system to the transformation $R$ is a possibility of existence there in-phase oscillations, which are satisfied condition $x=y$. Consequence of the symmetry of the system to the transformation $R \circ I$ is a possibility of existence there anti-phase oscillations, which are satisfied condition $x=-y$.

\section{INVESTIGATION OF THE IN-PHASE SYNCHRONIZATION IN THE SYSTEM}

In the system of coupled maps with changing of the controlling parameter $a$ transition to chaos occurs through the period-doubling bifurcations cascade. As a result near the points $1 C_{1}^{0}$ and $1 C_{2}^{0}$ chaotic attractors which relate to synchronous oscillations are formed. They locate in the subspace $x=y$. The Figure 1 demonstrates bifurcational lines which correspondent to bifurcations in the symmetric subspace: $l_{t d}^{1}, l_{t d}^{2}, l_{t d}^{4}, l_{t d}^{8}$ (the upper index is the periodicity of the orbit) are lines of period-doubling bifurcations of periodic orbits $C^{0}, 2 C^{0}, 4 C^{0}, 8 C^{0}$ (the first index denotes the periodicity of the orbit, the upper index is the time delay between oscillations of the subsystems) respectively. The largest multiplier becomes equal to -1 and correspondent eigenvector directs tangentially to the symmetric subspace. As a result these orbits become saddles and in their vicinity, in the same subspace stable periodic orbits of double periods appear. This cascade of sub-harmonic bifurcations leads to formation of a chaotic attractor in the symmetric subspace. This chaotic attractor relates to the regime of in-phase synchronization of chaos. Over the line $l_{t d}^{8}$ synchronous oscillations become chaotic. In the chaotic region under the line $l_{t m}^{2}$ (this line is the bound-merging bifurcation for the two-bound chaotic attractor) there are bound-merging bifurcations of chaotic attractors $2^{N} A^{0}$ which exist in the symmetric subspace. Over the line $l_{t m}^{2}$ in the subspace there are one-bound attractors $A_{1,2}^{0}$. On the line $l_{t u}^{1}$ these attractors unite and the united synchronous chaotic attractor is formed. Locations of the periodic orbits $C^{0}, 2 C^{0}$ and $4 C^{0}$ are presented in the Figure 2 for the values of the parameters: $a=3.65, \gamma=0.15$. The symmetric subspace is marked by dashed line.

Now, let's consider bifurcations which lead to appearance of new limit sets outside the symmetric subspace. On the line $l_{n d}^{1}$ (Fig. 1) the saddle fixed point $C^{0}$ undergoes the second period-doubling bifurcation. The second multiplier becomes equal to -1 and the correspondent eigenvector is directed normally to the subspace $x=y$. As a result the fixed point loses its stability in the normal to the symmetric subspace direction and becomes repeller. A saddle period-two orbit $2 C^{1}$ appears in its vicinity outside the symmetric subspace (Fig. 2). On the line $l_{p}^{2}$ its largest multiplier 


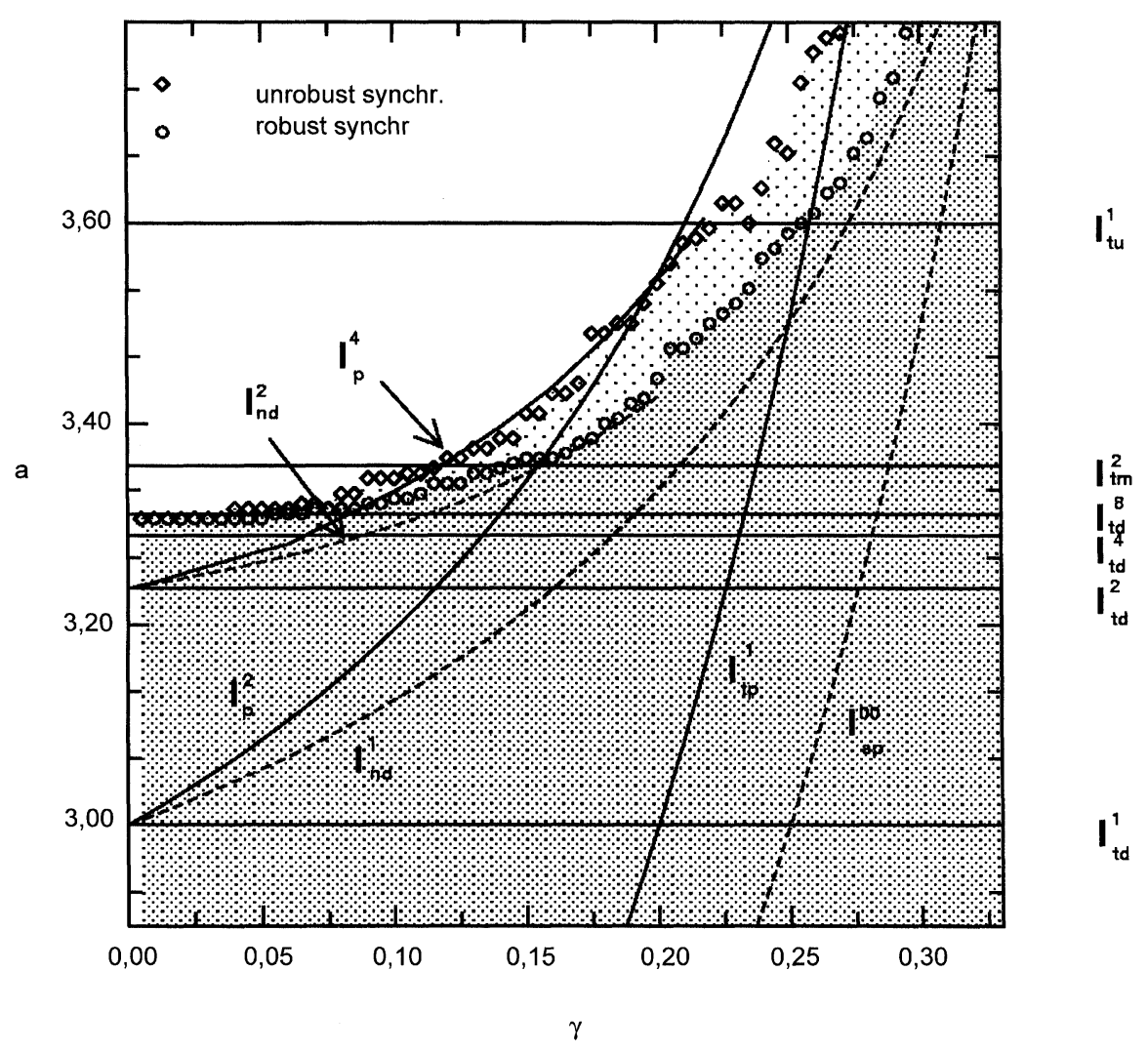

FIGURE 1 Bifurcational lines of symmetric periodic orbits and boundaries of unrobust $(\circ)$ and robust $(\diamond)$ in-phase synchronization of chaos on the pane of the parameters $\gamma-a$.

becomes equal to +1 (the pitchfork bifurcation), as a result this orbit becomes stable. On the line $l_{n d}^{2}$ the saddle period-two orbit $2 C^{0}$ undergoes the second period-doubling bifurcation. The second multiplier becomes equal to -1 . As a result the saddle becomes a repeller and in its vicinity outside the symmetric subspace a saddle period-four orbit $4 C^{2}$ (Fig. 2) appears. This orbit becomes stable on the line $l_{p}^{4}$.

On the line $l_{a p}^{00}$ the fixed point in the origin of the coordinates $C_{00}(x=0, y=0)$ loses its stability via the bifurcation of symmetry breaking (the multiplier is equal to +1 ) in another direction. As a result in its vicinity a pair of symmetric to each other saddle fixed points $C_{10} C_{20}$ appear, which are located in the other symmetric subspace $x=-y$ (we'll call it as "antisymmetric subspace"). On the line $l_{t p}$ these fixed points become stable.
Every second period-doubling bifurcation for periodic orbits $2^{N} C^{0}$ located in the symmetric subspace, which is accompanied by transformations their to repellers adds points of local transversal instability to the chaotic attractor $A^{0}$. From these points phase trajectory leaves the symmetric subspace at determine perturbations. For some systems it was demonstrated (Ashvin, 1994; Sushchik, 1997; Astakhov, 1997) that presence of such limit sets in the attractor leads to the bubbling phenomenon. In this case the regime of synchronous oscillations becomes unrobust. Any small noise and mismatch of the subsystems lead to destruction of the complete synchronization. The time-series of the difference $x_{n}-y_{n}$ is intermittency process (on-off intermittency), when motion in the symmetric subspace is intermittent by bursts from it. As a result a boundary of the 


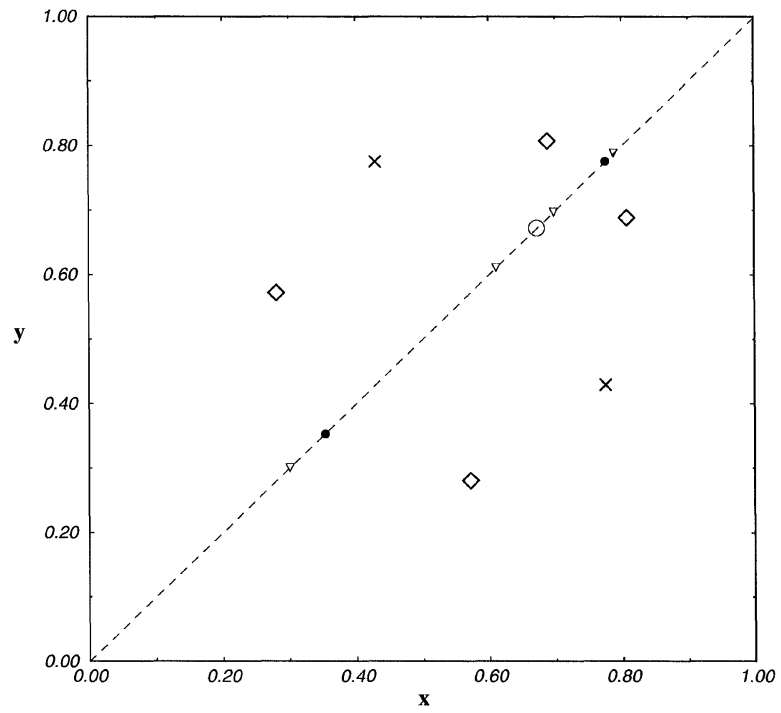

FIGURE 2 Location of periodic orbits taking part in the destruction of the in-phase chaotic synchronization: $C^{0}(\bigcirc)$, $2 C^{0}(\bullet), 4 C^{0}(\nabla), 2 C^{1}(\times), 4 C^{2}(\diamond)$.

synchronous region in the system with any small noise is shifted relatively to one in the system without noise. In our work we hold numeric investigations on determination of the boundary of the synchronization region. In these investigations we suppose oscillations as synchronous if the time-series of the subsystems are equal with precision of $\varepsilon$ during the whole time interval of observations:

$$
\max \left|x_{n}-y_{n}\right|<\varepsilon, n=1,2,3, \ldots, N_{\text {observ. }} .
$$

This value was chosen: $\varepsilon=0.0001, N_{\text {observ. }}=$ 2000000 iterations. In the Figure 1 (o) mark the experimentally determined boundary of the robust synchronization region (this region is marked by grey color). From the right side of it stable synchronous chaotic oscillations take place. Under the line $l_{t m}^{2}$ the system demonstrates many-band synchronous chaotic attractors $2^{N} A^{0}$, between the lines $l_{t m}^{2}$ and $l_{t u}^{1}$ there is one-band synchronous chaotic attractor $A^{0}$, over the line $l_{t u}^{1}$ there is a united synchronous chaotic attractor which appeared as a result of merging chaotic sets on base of the fixed points $C_{1}^{0}$ and $C_{2}^{0}$. Adding small noise with intensity $\sim 0.00001$ to the system doesn't lead to desynchronization.

From the left side of this boundary and from the right side of the boundary marked by $(\diamond)$ there is a region of unrobust synchronous chaos (this region is marked by light grey color). The synchronous regime is observed only in the absence of any noise. The transition process to this regime has the form of intermittency. Its duration depends on the chosen initial conditions. Adding very small noise to the system destructs the synchronous regime. The system demonstrates bubbling behavior. In the Figure 3 there are the phase portraits of chaotic attractor in the system without noise (Fig. 3a) and with noise (Fig. 3b). The Figure $3 \mathrm{c}$ demonstrates the time-series of the bubbling behavior.

In the region from the left side of the boundary $(\diamond)$ synchronous oscillations are absent. The phase trajectory transits to another stable oscillating regime, which exists in the system at these parameters values. Lower the point of crossing of the lines $l_{p}^{2}$ and $l_{p}^{4}$ the trajectory transits to the outof-phase orbit $2 C^{1}$. Over this point till the line $l_{t u}^{1}$ they are the orbits $4 C^{2}$ or $2 C^{1}$. Over the lines $l_{t u}$ and $l_{t p}^{1}$ in the case of the united chaotic attractor, with the losing of normal stability the trajectory can transit to one of the fixed points $C_{10,20}$ located in the antisymmetric subspace $x=-y$.

Near the line $l_{p}^{2}$ the phenomenon of riddled basins is observed in the system. In the nearest vicinity of the attractor $A^{0}$ there are regions from basins of other attractors. In the Figure 4 there is basins of the attractor $A^{0}$ which contains regions from the basins of the periodic orbit $2 C^{1}$ (black color). Adding of small noise destructs the basins of $A^{0}$, trajectory leaves to the orbit $2 C^{1}$ at any initial conditions near the $A^{0}$. But the time of the transient process depends essentially on the initial conditions.

Evidently the described picture of the region of synchronization is not detailed. The region contains also windows of periodicity and regions of attractors originated from these periodic regimes. Therefore the boundary of the synchronization 

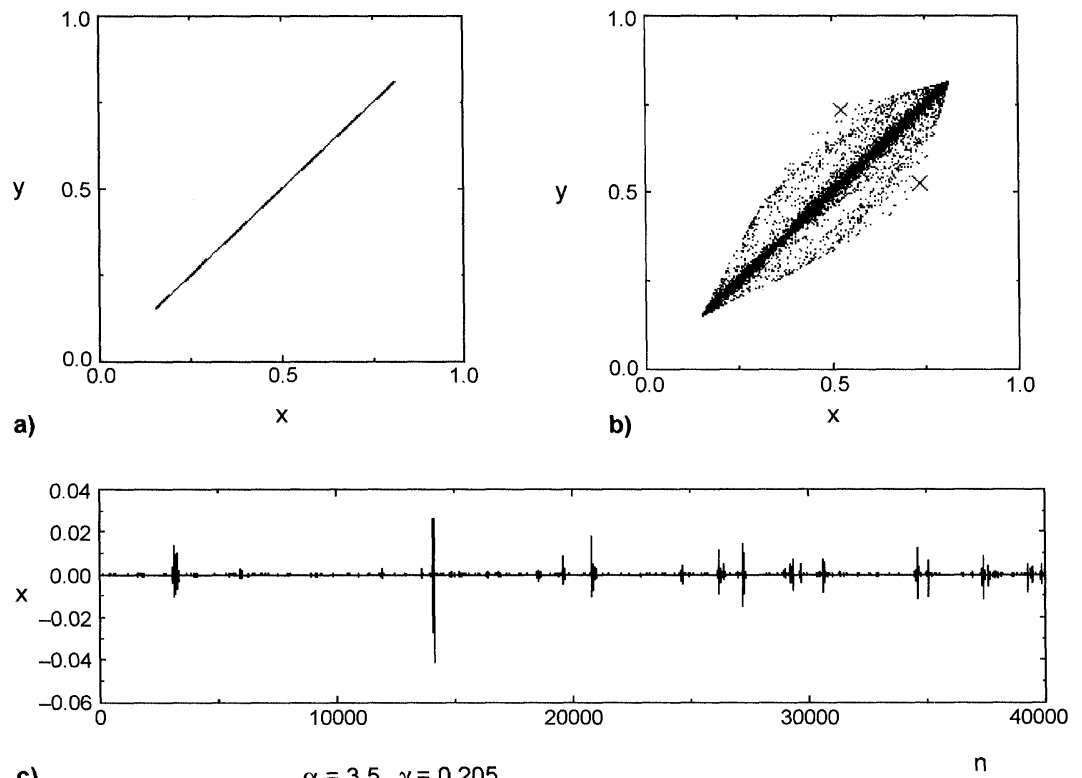

c)

$\alpha=3.5 \gamma=0.205$

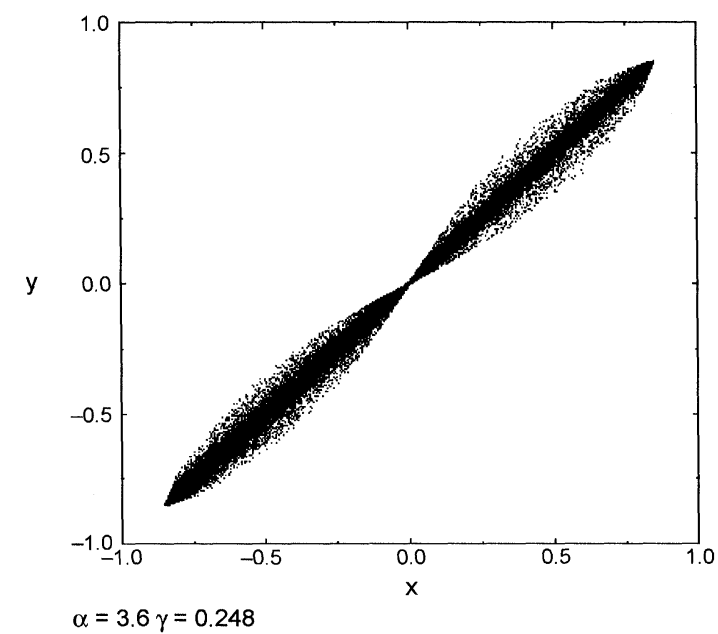

FIGURE 3 The phase portrait of the in-phase oscillations without noise (a) and with noise (b); time-series $x_{n}-y_{n}$ of the bubbling attractor (c). The phase portrait of the bubbling united chaotic attractor $(\mathrm{d})$.

region must be very complex. In our work we give the synchronization region only for chaotic attractors originated on the base of the main family of the periodic orbits $2^{N} C^{0}$.

Comparing experimental observations and results of the bifurcational analysis we can clear the mechanism of destruction of chaotic synchronization. Unlike some described cases (see for example (Sushchik, 1997; Astakhov, 1997) the lost of normal stability of the fixed point $C^{0}$ doesn't lead immediately to the bubbling attractor. The bubbling phenomenon appears at lower coupling, near the parameter points when other saddle orbits of the main family: $2 C^{0}, 4 C^{0}, 8 C^{0}, \ldots$ undergo the correspondent bifurcations. Hence, though in the chaotic attractor there are "defect points", bursts from the symmetric subspace are not observe in experiment for finite, but long time. 


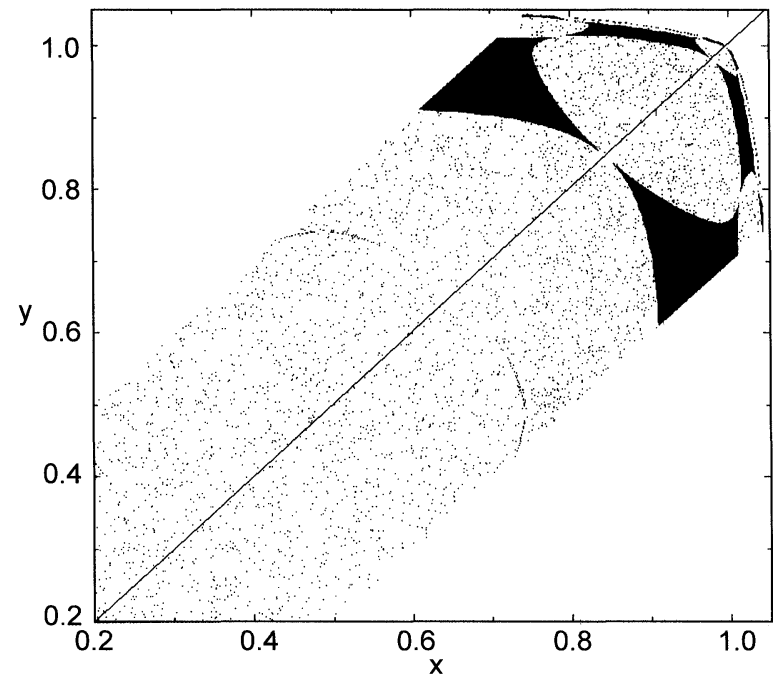

FIGURE 4 Basins of the chaotic attractor in the symmetric subspace (white color) and of the periodic orbit $2 C^{1}$ (black color). $a=3.5, \gamma=0.17$.

They appear after bifurcations of sufficient number of the other saddle orbits inside $2^{N} C^{0}$ the attractor.

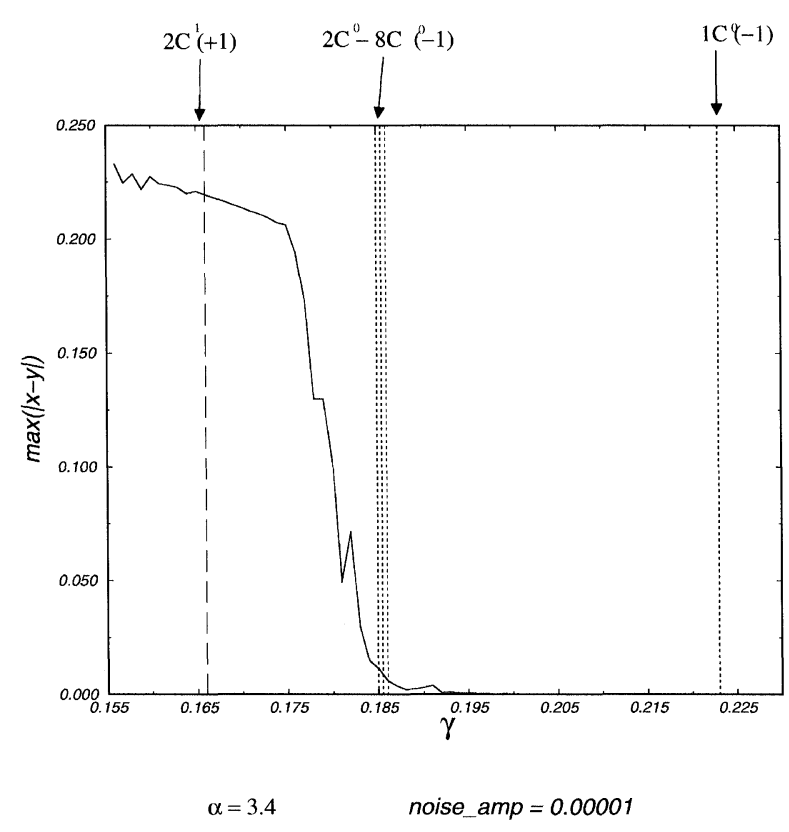

FIGURE 5 Transversal "size" of the bubbling attractor $\left(\max \left|x_{n}-y_{n}\right|\right)$ in dependence on the value of the coupling. $a=3.4$.
Transversal size of the appeared bubbling attractor is determine by the location of the points of the saddle periodic orbit $2 C^{1}$ (in the Fig. $3 \mathrm{~b}$ these points are marked by $\times$ ). Therefore this orbit and its manifolds must play important role in the formation of the bubbling attractor. The further decreasing of the coupling enforces the phenomenon of bubbling. The bursts appears more often and their amplitude increases. The dependence of the "thickness" of the bubbling attractor $\left(\max \left|x_{n}-y_{n}\right|\right)$ on the coupling coefficient is presented in the Figure 5. The parameter $a$ was chosen in the value 3.4. Oscillations in the system were investigated during interval of $\sim 10^{7}$ iterations. From the figure it is seen that the beginning of the bubbling appears until the points of bifurcations of the saddle orbits $2 C^{0}-8 C^{0}$ but these bifurcations enforce this phenomenon.

\section{ANTIPHASE REGULAR REGIMES AND CONTROLLED ANTIPHASE CHAOTIC SYNCHRONIZATION}

Antiphase regimes are formed on the base of the fixed points $C_{10}$ and $C_{20}$. Limit sets formed on the base of these points are identical up to symmetry transformation. Therefore we'll consider only one family of the regimes (for example, near the point $C_{10}$ ).

The saddle fixed point $C_{10}$ appears from the saddle fixed point $C_{00}$ in the result of the symmetry breaking bifurcation. It is unstable to the perturbations directed transversally to the antisymmetric subspace. On the line $l_{t p}^{1}$ (Fig. 6) it becomes stable through pitchfork bifurcation. With changing of the parameters $a$ and $\gamma$ on the base of this fixed point there is a cascade of the period doubling bifurcations which leads to formation of a chaotic set inside the antisymmetric subspace. The every orbit undergoes the period-doubling bifurcation twice in the cascade. Firstly, as stable orbit on the first multiplier, secondly as saddle orbit on the second multiplier. As a result of the first period doubling the orbit loses its stability in the normal 


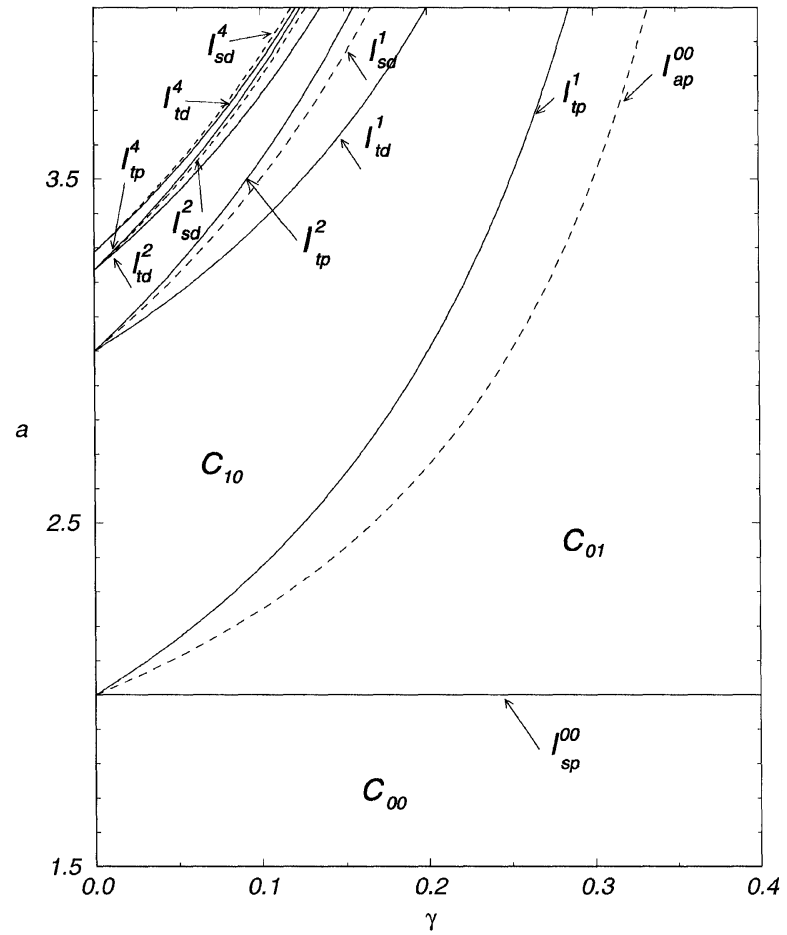

FIGURE 6 Bifurcational lines of antisymmetric periodic orbits on the pane of the parameters $\gamma-a$.

to the subspace direction. In its vicinity, outside the antisymmetric subspace a stable orbit of double period appears. As a result of the second period doubling the saddle orbit loses stability in the tangent direction and becomes repeller. In its vicinity, inside the antisymmetric subspace a saddle periodic orbit of double period appears. In the Figure 6 the lines of the first period doubling bifurcations are denoted: $l_{t d}^{1}$ (for the orbit of the period-one), $l_{t d}^{2}$ (for the orbit of the period-two), $l_{t d}^{4}$ (for the orbit of the period-four), and the lines of the second period doubling bifurcations as $l_{s d}^{1}, l_{s d}^{2}, l_{s d}^{4}$ respectively. Then, with further parameters changing, the appeared saddle antisymmetric orbits become stable through the subcritical pitchfork bifurcations. In the Figure 6 these lines are denoted as $l_{t p}^{2}$ and $l_{t p}^{4}$. Therefore, on the parameters plane in the region between the lines $l_{t p}^{1}$ and $l_{t d}^{1}$ there is a stable antiphase period-one orbit, between the lines $l_{t p}^{2}$ and $l_{t d}^{2}$ there is a stable antiphase period-two orbit and between the lines $l_{t p}^{4}$ and $l_{t d}^{4}$ there is a stable antiphase period-four orbit. We didn't consider bifurcations of the orbits of larger periods but one can conclude that they occur by the same way. Hence, on the plane of the parameters there are bounds of stability for regular antiphase regimes, between which bounds of transversal instability exist.

The considered bifurcational scenarium is very similar to the one for in-phase orbits. However, in the case of in-phase synchronization the bifurcations inside the symmetric subspace precede the bifurcations in the normal direction. Therefore, in the case of antiphase synchronization, contrary to the in-phase synchronization:

- regions of transversal stability are divided by the regions of transversal instability;

- in the symmetrical subspace the transversally stable chaotic attractor is not formed.

To stabilize previously unstable regime of antiphase chaotic synchronization we used the controlled term $r(f(x)+f(y))$ which was added to the right side of the first equation of the system (19):

$$
\begin{aligned}
x_{n+1}= & (a-1) x_{n}-a x_{n}^{3}+ \\
& \gamma\left[(a-1) y_{n}-a y_{n}^{3}-(a-1) x_{n}+a x_{n}^{3}\right] \\
+ & r\left(f\left(x_{n}\right)+f\left(y_{n}\right)\right) \\
y_{n+1}= & (a-1) y_{n}-a y_{n}^{3}+ \\
& \gamma\left[(a-1) x_{n}-a x_{n}^{3}-(a-1) y_{n}+a y_{n}^{3}\right]
\end{aligned}
$$

Choosing $r$ is close to -1 it is possible to make the normal Lyapunov exponent sufficiently small and hence, the synchronous chaotic regime transversally stable. In the Figure $7 \mathrm{a}$ we plotted the dependences of the normal Lyapunov exponent on the controlling parameter $r$. Values of the other parameters corresponds to the regime of the united chaotic attractor: $a=3.8, \gamma=0.04$. To transit to the regime of antiphase synchronization we used the following procedure: We chosen initial conditions from the basins of the united chaotic attractor. In the every moment of time we appreciated the distance between the phase point 


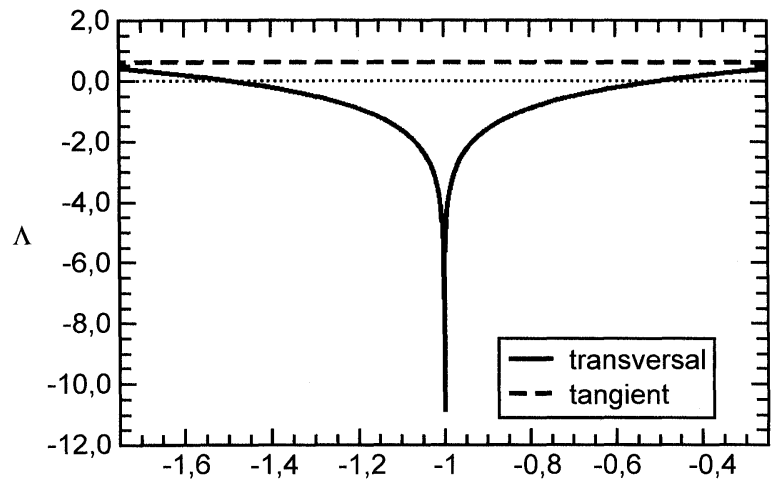

a)

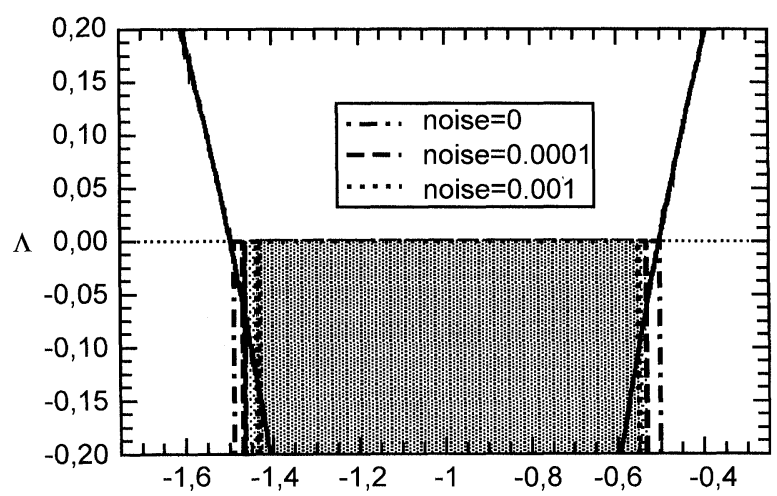

b)

FIGURE 7 Dependence of the normal Lyapunov exponent for antiphase chaotic attractor on the controlling parameter $r$ (a) at $a=3.8, \gamma=0.04$. Dashed line denotes the values of the tangent Lyapunov exponent. In the (b) this dependence is presented in larger scale with regions of the antiphase controlled synchronization.

and the antisymmetric subspace: $\rho=\left|x_{n}+y_{n}\right|$. The distance was compared with the chosen value $\delta$. If $\rho>\delta$, the phase point is far from the subspace and the controlling influence is switched off. The trajectory evolves on the unsynchronous chaotic attractor. When phase point appears near the antisymmetric subspace $(\rho \leq \delta)$, the controlling influence is switched on. If the controlling parameter $r$ locates in the interval where $\Lambda_{\perp \text { contr }}<0$ the chaotic set inside the antisymmetric subspace becomes stable to transversal perturbation and the trajectory is attracted to the subspace. After this the controlling influence tends to zero. In our numerical experiments we chose $\delta=0.01$. In the
Figure 8 phase portraits of the oscillations without control (a), with control (c,e) and correspondent time-series of $x_{n}+y_{n}(\mathrm{~b}, \mathrm{~d}, \mathrm{f})$ are presented. The original chaotic attractor (Fig. 8a) corresponds to the regime of awfully unsynchronous chaos. The phase trajectory draws the square like region. With applying small controlling influence the diagonal line $x=-y$ appears on the region (Fig. 8c). The time-series has interval of synchronous behavior (Fig. 8d). With further changing $r$ the intervals of synchronous behavior grow and as a result the system transits to awfully synchronous oscillations (Fig. 8e,f). In this case the resulting chaotic attractor is a one-band attractor located in the antisymmetric subspace. In the Figure $7 b$ the intervals of the parameter $r$ sufficient for complete synchronization at different intensities of noise are presented. The more dark color corresponds to larger noise. Without noise the interval of the synchronization coincides with the interval of $r$ where the normal Lyapunov exponent is negative. With noise the controlled synchronization region becomes more narrow (Fig. 7b).

The process of the in-phase synchronization loss is accompanied by the bubbling phenomenon and riddled basins. It is reasonable question: Do these phenomena exist in the case of antiphase synchronization loss. To answer this question we considered the evolution of the chaotic attractor with changing of the coefficient $r$. In the numerical experiments we chose initial values near the antisymmetric subspace and the controlling influence was switched on not depending on nearness $\rho$ of the phase point to the subspace. At $-1.46<r<-0.525$ the chaotic attractor inside the antisymmetric subspace is stable to transversal perturbations. The synchronous regime is robust. Adding noise of small intensity $(\sim 0.00001)$ doesn't lead to visible changing in the system's behavior. With increasing of the controlling parameter at $r>-0.525$ a bubbling attractor is observed in the system. The chaotic attractor remains stable to transversal perturbations but the time of transient process becomes extremely large (hundreds of thousands iterations) and it sensibly 

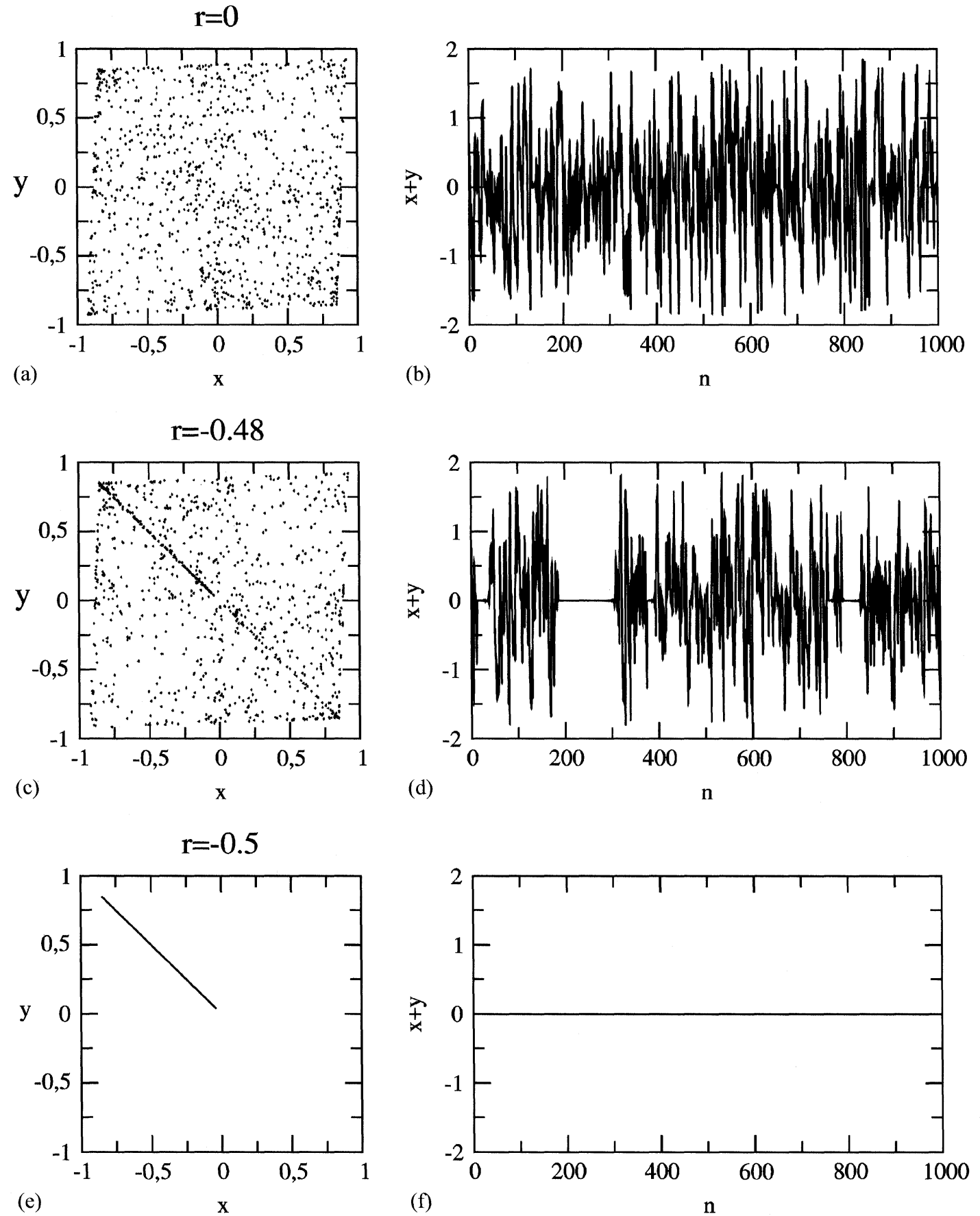

FIGURE 8 The phase portraits and time-series of the oscillating regimes without control (a,b), with partial control (c,d) and in the regime of the complete antiphase synchronization $(e, f)$.

depends on the initial values. Adding noise of small intensity leads to essential rebuilding of the phase portrait of the oscillations. The attractor gets finite transversal size. Phase point begins to visit neighborhoods of the both fixed points $C_{10}$ and $C_{20}$. The corresponding time-series of $x_{n}+y_{n}$ is 

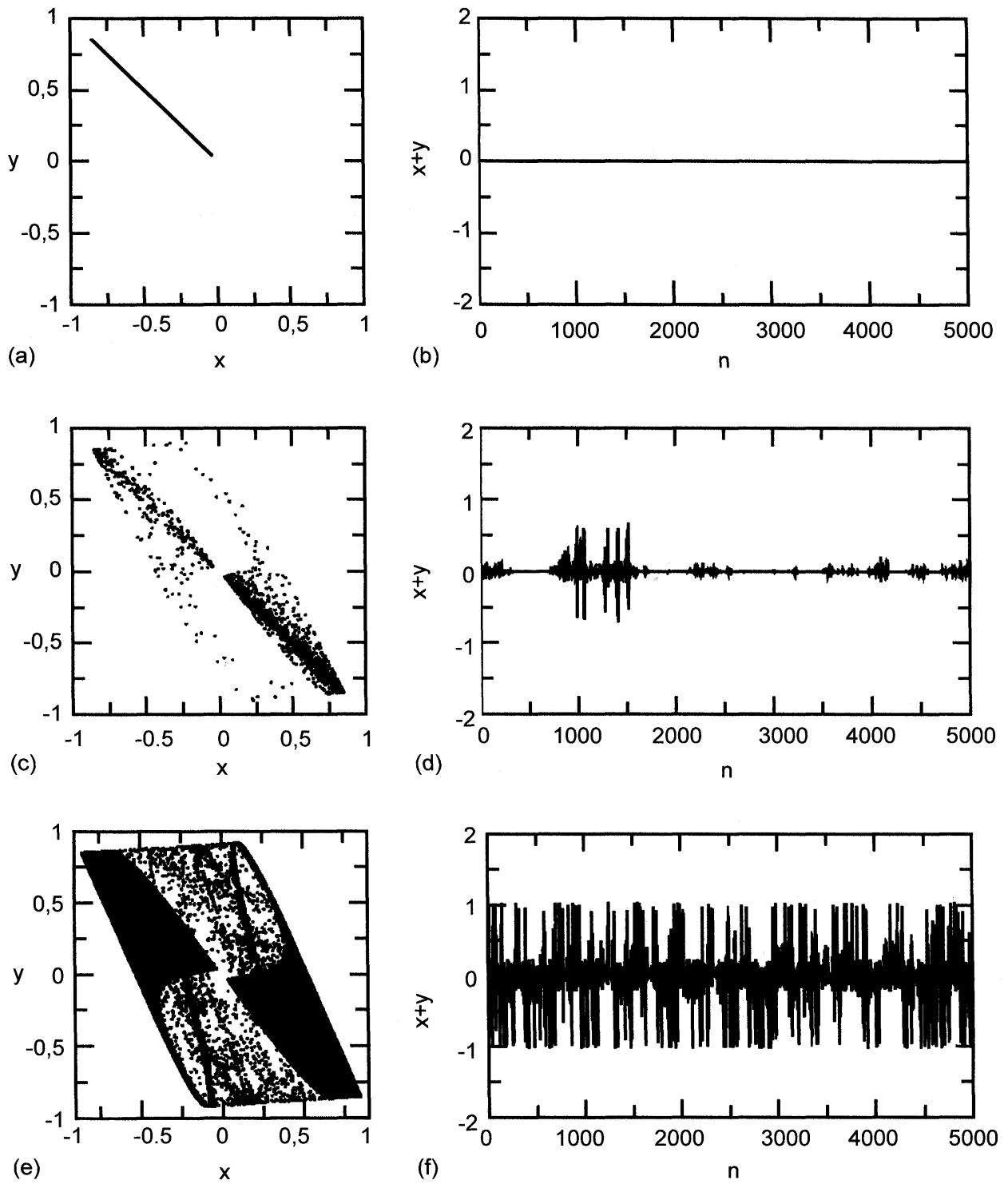

FIGURE 9 The phase portraits and time-series of antiphase oscillations without noise (a,b) and with noise (c,d). In (e,f) there is chaotic oscillations resulted from the blowout bifurcation.

the on-off intermittency process (Platt, 1993). The Figure 9 presents phase portraits of the attractor without noise (a) and with small noise (c). In the (b,d) there are corresponding time-series. With small noise phase point moves along the antisymmetric subspace for a long time. Then, it is short burst apart from the subspace, after which the phase point return to the vicinity of the antisymmetric subspace. The averaged frequency of the bursts increase with increasing of the parameter $r$. Finally, at $r=-0.406$ the blowout bifurcation (Ashvin, 1994) takes place when the chaotic attractor is not already stable in the normal direction and it transforms to the chaotic saddle. The synchronous oscillations are not observed further in the system both with noise and without it. The phase portrait of oscillations looks like the bubbling attractor in presence of noise (Fig. 9e). 


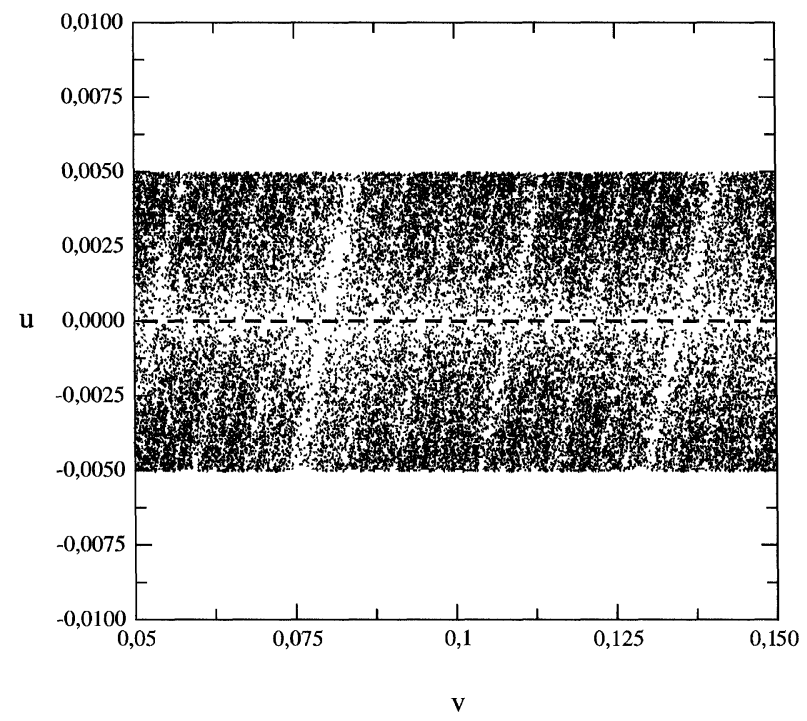

FIGURE 10 A part of the basins of the chaotic attractor inside the antisymmetric subspace (white color) at $r=-1.485$. The black color denotes regions relating to the basins of the attractor in the infinite.

With decreasing $r$ from the synchronization region, at $r<-1.46$ we observed the transition from the robust stable synchronous regime to the bubbling behavior. Then, at $r<-1.472$ the basins of the synchronous attractor is riddled by holes of the basins of the attractor in the infinity. In the Figure 10 we present a fragment of the basins of the chaotic attractor in the antisymmetric subspace (white color) with holes from the basins of the infinity attractor (black color) wedged in it. This basins is represented in the normal coordinates $u$ and $v$, the antisymmetric subspace is marked by the dashed line. The results were obtained for the parameters values: $a=3.8, \gamma=0.04, r=-1.485$.

\section{CONCLUSION}

We considered in-phase and antiphase complete chaotic synchronization in the system of coupled cubic maps. The in-phase self-synchronization of chaos is possible for sufficiently large coupling coefficient. We investigated bifurcational mechanisms which lead to destruction of the regime of robust synchronization in a system of coupled cubic maps. As for other coupled systems with period-doublings, exit from the regime of synchronous chaos is accompanied by the bubbling of the attractor and then by the riddling of its basins. Our investigations of coupled logistic maps (Astakhov, 1997; Astakhov, 1998) and this work demonstrate that bifurcations of saddle orbits of the main family $2^{N} C^{0}$ inside the attractor play the main role in this process. Nevertheless, unlike the other similar systems, experimentally observed exit from the regime of robust synchronization takes place at smaller value of the coupling that the first bifurcation of the saddle orbit in the attractor.

We demonstrated that bifurcational mechanisms inside the antisymmetric subspace are similar to ones in the symmetric subspace except the order of bifurcations taking place tangiently and normally to the subspace. The antiphase self-synchronization in similar systems is possible only for regular regimes. The chaotic synchronization can be achieved with applying methods of chaos control. In the work we used the control method for antiphase chaotic synchronization in the form of additional feedback loop. We demonstrated that the process of loss of this type of synchronization can be similar to the case of in-phase synchronization. It demonstrates bubbling behavior, riddled 
basins and blowout bifurcation. The bifurcational mechanisms of the loss of antiphase synchronization is the subject for future consideration.

\section{Acknowledgments}

This material is based upon work supported by the Naval Research Laboratory under Contract No. N68171-00-M-5430. The authors also thanks to the Fond of Civil Research Development (Grant REC 006) and Russian Fond of Basic Researching (Grant 00-02-17512) for partial finanical support.

\section{References}

Ashvin, P., Buescu, J. and Stewart, I. (1994) "Bubbling of attractors and synchronization of chaotic oscillators", Physics Letters A, 193, 126-139.

Ashvin, P., Buescu, J. and Stewart, I. (1996) "From attractors to chaotic saddle: a tale of transverse instability", Nonlinearity, 9, 703-737.

Astakhov, V., Shabunin, A., Kapitaniak, T. and Anishchenko, V. (1997) "Loss of chaos synchronization through the sequence of bifurcations of saddle periodic orbits", Physical Review Letters, 79(6), 1014-1017.

Astakhov, V., Hasler, M., Kapitaniak, T. Shabunin, A. and Anishchenko, V. (1998) "Effect of parameter mismatch on the mechanism of chaos synchronization loss in coupled systems", Phys. Rev. E, 58(5), 5620-5628.
Cao, L.-Y. and Lai, Y.-C. (1998) "Antiphase synchronism in chaotic systems", Phys. Rev. E, 58, 382-386.

Fujisaka, H. and Yamada, T. (1983) "Stability theory of synchronized motion in coupled-oscillator systems", Progress of Theoretical Physics, 69, 32-47.

Fujisaka, H. and Yamada, T. (1985) "A new intermittency in coupled dynamical systems", Progr. Teor. Phys., 74(4), 918-921.

Kuznetzov, S. P. (1985) "Universality and similarity in behavior of coupled Feigenbaum systems", Radiophizika, 28(8), 991.

Lai, Y.-C., Grebogi, C., Yorke, J. A. and Venkataramani, S. C. (1996) "Riddling bifurcation in chaotic dynamical systems", Phys. Rev. Lett., 77(1), 55-58.

Maistrenko, Y. L., Maistrenko, V. L. and Popovich, A. (1998) "Transverse instability and riddled basins in a system of two coupled logistic maps", Phys. Rev. E, 57(3), $2713-2723$.

Maistrenko, Y. L., Maistrenko, V. L., Popovich, A. and Mosekilde, E. (1999) "Desynchronization of chaos in coupled logistic maps", Phys. Rev. E, 60(3), 2817-2830,

Pecora, L. M. and Caroll, T. L. (1990) "Synchronization in chaotic systems", Phys. Rev. Lett., 64, 821-824.

Pikovsky, A. S. and Grassberger, P. (1991) "Symmetry breaking bifurcation for coupled chaotic attractors", J. Phys. A: Math., 24, 4587-4597.

Pikovsky, A., Osipov, G., Rosenblum, M., Zaks, M. and Kurths, J. (1997) "Attractor-repeller collision and eyelet intermittency at the transition to phase synchronization", Physical Review Letters, 79.

Platt, N., Spiegel, E. A. and Tresser, C. (1993) "On-off intermittency: a mechanism for bursting", Phys. Rev. Lett., 70, 279-282.

Sushchik, M. M., Rulkov, N. F. and Abarbanel, H. D. I. (1997) "Robustness and stability of synchronized chaos: an illustrative model", IEEE Transactions on circuits and systems, 44(10), 866-873. 


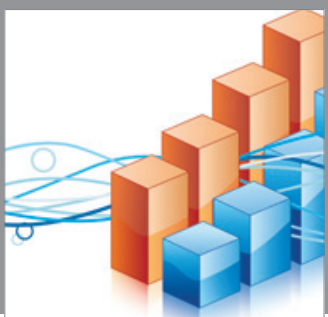

Advances in

Operations Research

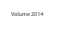

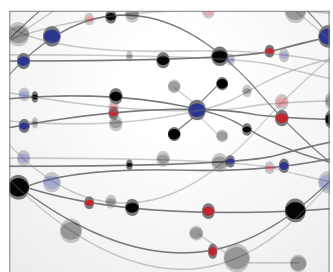

\section{The Scientific} World Journal
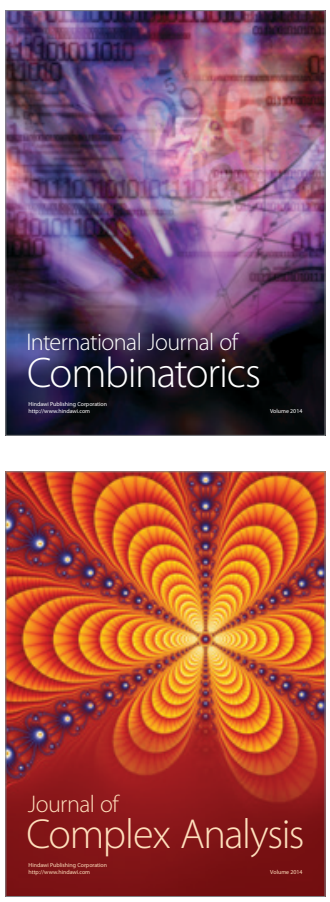

International Journal of

Mathematics and

Mathematical

Sciences
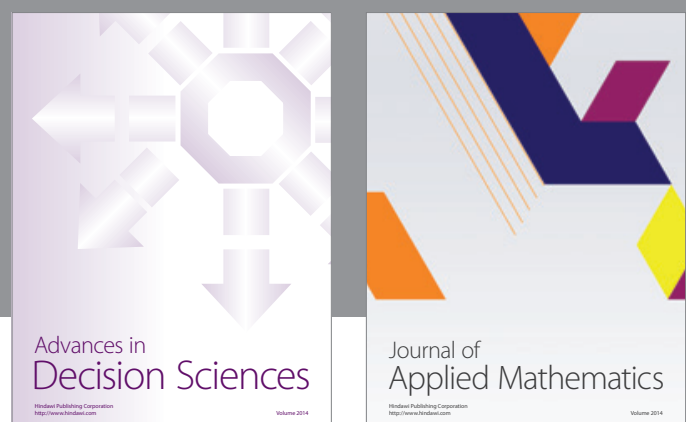

Journal of

Applied Mathematics
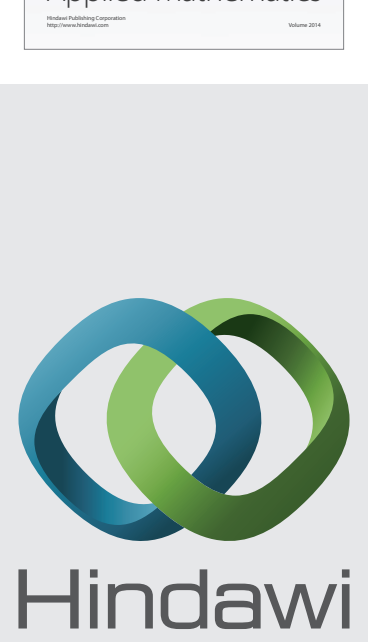

Submit your manuscripts at http://www.hindawi.com
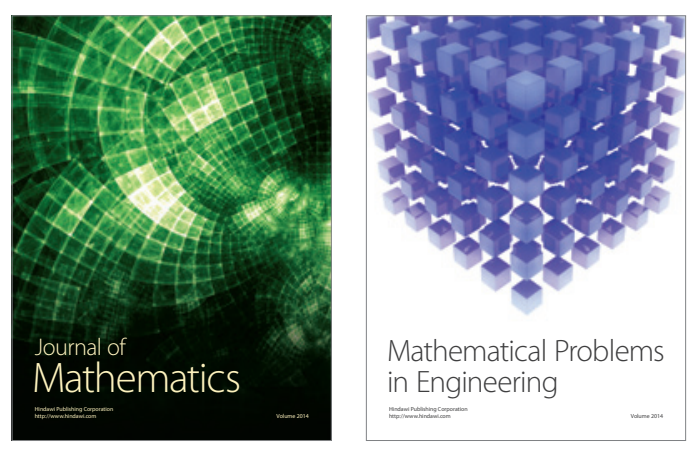

Mathematical Problems in Engineering
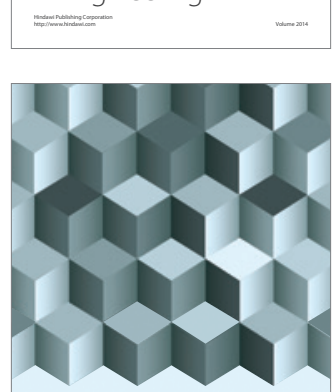

Journal of

Function Spaces
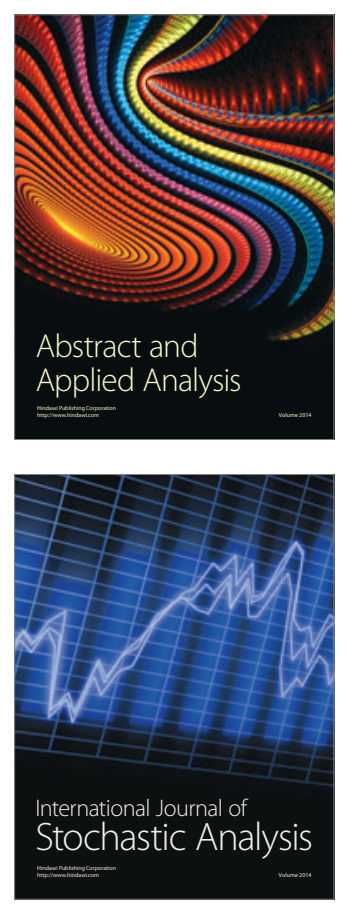

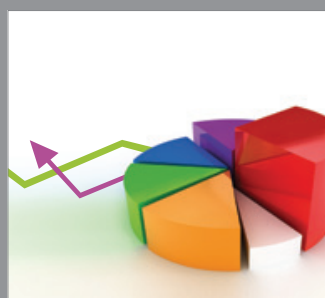

ournal of

Probability and Statistics

Promensencen
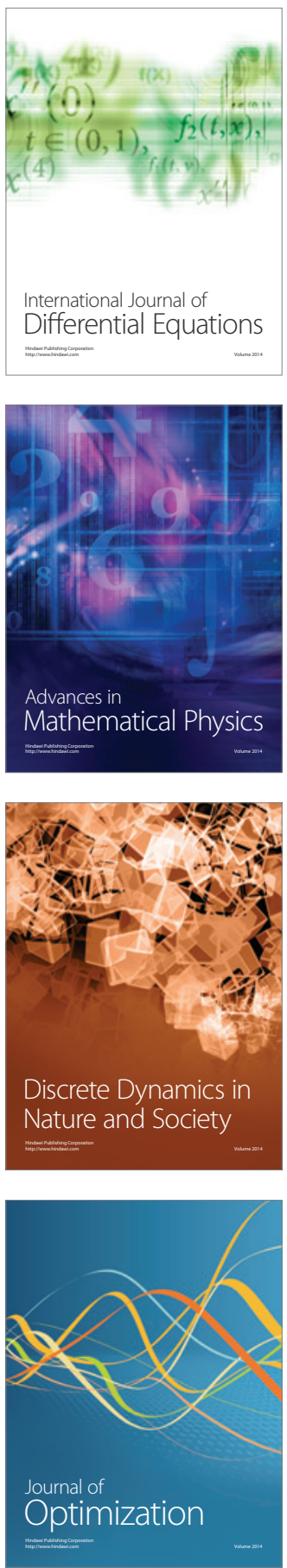